\title{
Design and Realization of Convergence Controller Based on IEC61850 Protocol
}

\author{
Fengzhen Zhou, Yufeng Duan, Ningxi Song and Jingchao Zhang \\ State Grid Henan Electric Power Company, Henan Hebi Power Supply Company \\ Zhengzhou, China \\ Email: zhoufengzhen@sgepri.sgcc.com.cn
}

\begin{abstract}
By deploying a variety of intelligent devices to the smart grid, the power Internet of Things could achieve safe and reliable information transmission, collaborative processing, unified services and application integration, help promote the panoramic awareness, interoperability and seamless integration in the whole process of power grid operation and enterprise management. The increasing of number and types of sensors will result in the parallel use of variety sensing technologies and specifications, therefore, it's an urgent need for research and realization of convergence controller which supports the IEC61850 protocol, providing communication interfaces and specifying accesses of different protocols, in order to achieve convergence, agent and procession of the large number of sensing data.
\end{abstract}

Keywords-Convergence controller; IEC61850; software component; hierarchical structure

\section{INTRODUCTION}

With the fast development of power grid in china, intelligent equipment and systems are becoming increasingly complex and large for years. ${ }^{[1]}$ On one hand, it is necessary for development of various types of sensing devices to achieve perception and collection of site information and improve the depth and breadth of information sources; on the other hand, the increasing number and types of sensors will result in the use of various sensing technologies and specifications which brings out the non-unified expression of various collection data (insists of identity, semantics, data presentation formats, etc.). Convergence controller is mainly deployed in the substation for aggregation, processing and transferring the monitoring data uploaded by monitoring devices. Considering the IEC61850 has been widely used as the sole specification in substation communication networks and systems, therefore, it's an urgent need for research and realization of convergence controller that supports the IEC61850 protocol and provides communication interface as well as specified access for different protocols, in order to achieve the function of summary and arrangement of the large number of sensing data, avoiding network congestion and other problems. After been analyzed and processed by the convergence controller, real-time monitoring data of various types of sensors will be mapped to MMS (Manufacturing Message Specification) service uniformly, in order to be real-time received by clients that use IEC61850 as communication protocol in the application layer. ${ }^{[2]}$

Based on the demands of application and recent research fruits, this paper systematically analyses and designs the technical route, overall architecture, modeling tools, IEC61850 server functionality, and compatible communication adapters of the convergence controller.

\section{Technical Route Of The Convergence CONTROLLER}

As the first layer of the convergence controller software; the OS (Operation System) will affect the performance of convergence controller directly. In power communication system, embedded real-time operating system is generally chosen to meet the real-time requirement.

The embedded Linux OS is customized designed, based on the self-developed green communication server. Linux OS provides a user-controllable, priority-driven and urgent priority preemptive scheduling mode. ${ }^{[3-4]}$

The convergence controller software takes GCC \& QT as development platform. GCC is not only the most important software development tool, but also the most commonly used compiler. As a compiler that meets the ANSI C standard in the GNU project, it can compile programs of $\mathrm{C}, \mathrm{C}++$, Object $\mathrm{C}$, etc. GCC is not only very powerful, but also has a very flexible structure, through which programmers are able to control the entire compilation process better. QT is a cross-platform development framework for C++ GUI applications.

Object-oriented programming and software component technology is applied in building the application software in the convergence controller. Communication protocol management consists two parts: main scheduler of the convergence controller and communication protocol manager. The main scheduler of the convergence controller manages advanced applications, while the communication protocol manager manages the access of communication protocol.

\section{Overall Architecture Of The CONVERgence}

Based on the IEC61850 smart device model, this paper describes electrical equipment with SCL language and establishes a unified information modeling tools. For monitoring devices which comply with the standard communication protocol and unified information model, direct access is allowed thought IEC61850 communication adapter (IEC61850 client module). If existing monitoring devices do not comply with the standard communication protocol, communication adapters are designed and developed for corresponding device to complete the 
conversion of communication protocol and information model.

The convergence controller provides the function of standard server based on IEC61850 and provides unified external data communication services based on IEC61850.

\begin{tabular}{|c|c|c|c|}
\hline \multicolumn{4}{|c|}{ IEC61850 Server } \\
\hline $\begin{array}{l}\text { Monitoring data } \\
\text { reading module }\end{array}$ & \multirow{2}{*}{\multicolumn{2}{|c|}{$\begin{array}{c}\text { Convergence } \\
\text { Controller }\end{array}$}} & Modeling tool \\
\hline $\begin{array}{l}\text { Monitoring data } \\
\text { storage module }\end{array}$ & & & $\begin{array}{l}\text { Monitoring data } \\
\text { configuration } \\
\text { module }\end{array}$ \\
\hline \multicolumn{4}{|c|}{ Monitoring data intelligent processing module } \\
\hline \multicolumn{2}{|c|}{ IEC61850 communication adaptor } & \multicolumn{2}{|c|}{$\begin{array}{c}\text { Private standard } \\
\text { communication adaptor }\end{array}$} \\
\hline \multicolumn{2}{|c|}{ Ethernet } & \multicolumn{2}{|c|}{ Communication medium } \\
\hline $\begin{array}{c}\text { IEC61850 Server } \\
\text { monitor No. } 1\end{array}$ & $\begin{array}{l}\text { IEC61850 Server } \\
\text { monitor No. } 2\end{array}$ & & $\begin{array}{l}\text { Private standard } \\
\text { monitor }\end{array}$ \\
\hline
\end{tabular}

Fig. 1. Overall architecture of convergence controller

\section{Modeling Tools Of The Convergence CONTROLLER}

IEC61850 defines the hierarchical structure of the substation model and the corresponding SCL also applies hierarchical structure to describe the data structure of substation. ${ }^{[4]}$ Structural model of SCL is shown in Figure 2.

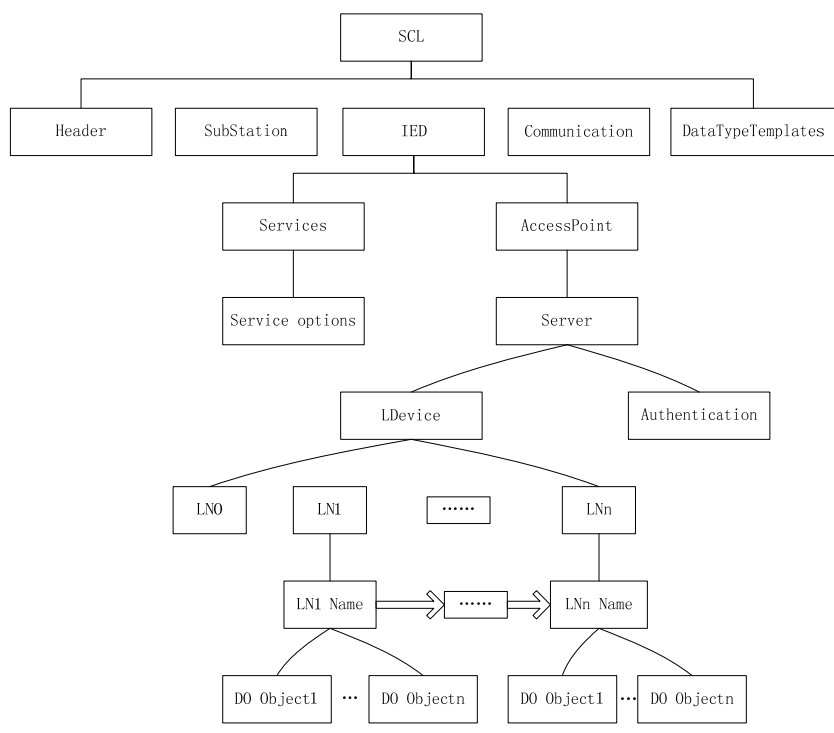

Fig. 2. SCL structure mode

This hierarchical model based on SCL files simulates this data structure by mesh data model, and extracts information from SCL files though XML parsing technology based on Microsoft's MSXML. Using nested chart, data, property and their relationship in each hierarchical can be shown and SCL document can be configured in a friendly human machine interface. The program is structured by $\mathrm{C} / \mathrm{S}$ mode and supports multiple IED reading and configuring. Plug-in technology architecture could be adopted in some detail parts (eg.XML module) in order to make use of the mature software modules which can benefit for future maintenance and upgrades. ${ }^{[5]}$

By applying modular design, component technology based on COM and using mature and open program module extensively, the convergence controller can reduce the development cost and time, and easy to be maintained and upgraded. The convergence controller design mainly consists of configuration interface module, XML parsing module, LN and CDC information library module, chart control module and SCL document import and export module.

\section{A. Configuration interface module}

This module mainly provides a visual friendly user configuration interface to open the related SCL file in format of IED, ICD etc. User can click on the menu or button "Configuration" to open the user configuration interface, then click on the corresponding property page to configure IED network parameters, data sets, GOOSE and other system requirements.

\section{B. XML parsing module}

The design selects the Microsoft's MSXML as parser. MSXML parser provides four different functions: the analyzer based on the Document Object Model (DOM), SAX (Sample API for XML) analyzer, XSLT processor, validating analyzer. Considering that the definition of IEC61850 substation data object model is based on a hierarchical model system, whose hierarchical relationship is logically a tree and three-dimensional, so the DOM parser is selected in the design of SCL configuration tool to operate this tree data structure more easily.

\section{LN and CDC information repository module}

$\mathrm{LN}$ and CDC information repository is mainly used to store some common data templates defined in IEC61850, such as the common data class information defined partly in IEC61850-7-3, compatible logic class information defined partly in IEC61850-7-4, and enumerated data information, etc. According to the understanding of protocols and configuration rules of the SCL document, the information repository can be defined by four data templates: LNodeType, DOType, DAType, and EnumType. ${ }^{[6]}$

\section{Chart control module}

For the hierarchical model, the tree control is an advanced programming technique at present for controlling and displaying, which can visually display the data hierarchies, but it can't visually display the relationships among the data and their attributes. The chart control model not only can display the association three-dimensionally and visually by using the tables and graphical markers, but also can add, delete and modify SCL element nodes effectively. The module can establish the mesh association model according to the data information acquired by XML module and display them by Crystal Cell, each hierarchy can use a relatively independent (each SCL file is a large table, the data is nested stored in the corresponding cell of the big table) table to show all of its data elements, and determine the number of rows and columns according to the number of 
data elements and data attributes automatically. The data of the same hierarchical layer is displayed in one row.

\section{E. Import and export modules for SCL documents}

One of the important function of modeling tools is importing a variety kinds of SCL files, and exporting configured SCL files. Import module calls parsing module to insert the node information of the SCL file into the SCL tree. Export module calls parsing module to save one or more nodes of the SCL tree as SCL file in XML format. The advanced system configurator will check grammar and show the errors, if exists, when importing a SCL file. The check accuracy and prompt friendliness are yet to be further improved, and the semantic check is also very few involved. When importing a SCL document of Chinese context, character encoding is also a problem, some use UTF8 encoding, some use GB2312 encoding, and others use GB18030 encoding, etc.

\section{IEC61850 SERVER FUNCTION}

The main function of IEC61850 server is to read the model profiles, analyze model and generate MMS types object space dynamically, which is also the type source of a variety of MMS Named Variable object. ${ }^{[7]}$

(1)The server parses the configuration file to generate MMS Domain object space dynamically according to the LD configuration of SCL.

(2)The server parses the configuration file to generate MMS Named Variable instance object space by LN units dynamically according to the LN configured of SCL.

(3)The server parses the configuration file to generate data-set and RCB corresponding object space according to the LNO's data-set configuration of SCL.

(4)The server constructs supporting system of MMS Log and MMS File object.

(5)Getting MMS objects based on the parsed above, the real-time database is created in server.

According to the mapping relationships, the data elements (temperature, humidity, etc.) in real-time database is updated correspondingly.

After received the of the client's request for report control blocks, the server checks whether the request is legitimate (whether the requested report control blocks exist or whether the total count have reached the maximum client capacity, etc.), if the request is legitimate, a signal confirm of request promised is send to the client and the server is ready to generate data message for the corresponding client. There are two ways to send packets, one way has cache and the other not.

The server can provide reporting services to multiple clients simultaneously.

The server supports a variety of services within the IEC61850 protocol. ${ }^{[8]}$

The basic data process of IEC61850 server program is shown in Figure 3 blow.

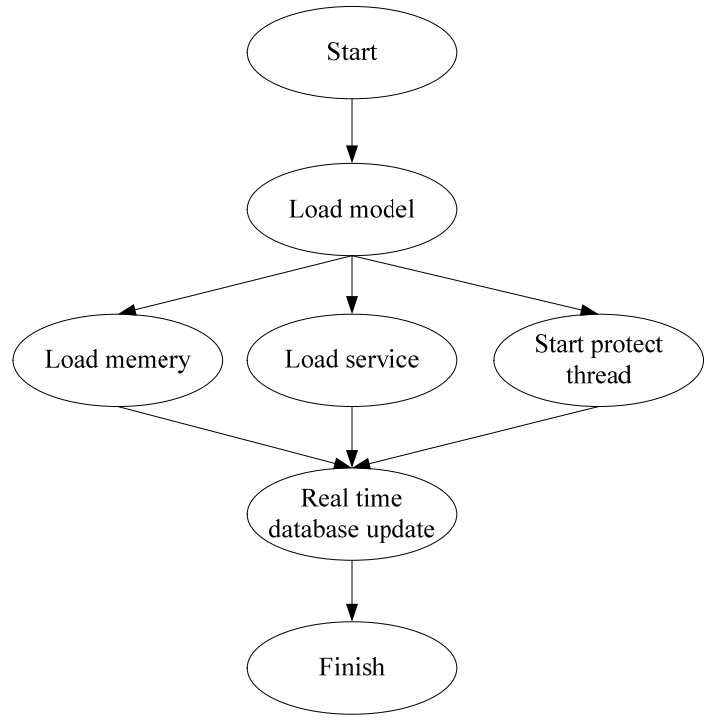

Fig.3. Basic process of IEC61850 server

\section{COMMUNICATION ADAPTER DESIGN}

Communication adapter is implemented as a dynamic link library: Each protocol is encapsulated into a dynamic link library, called by the main program of the convergence controller. Thus, the device meets not only the old access, but also monitoring device's data access, which is designed and developed according to IEC61850 protocol.

Call rules:

(1) Read the communication configuration information of all stations monitoring device from the list of devices in the database.

(2) According to the different ways of communication, start the corresponding dynamic link library to connect the equipment, the corresponding relationship: communication hardware environment - corresponding device corresponding standard - corresponding dynamic link library. If more than one device has the same communication environment, a dynamic link library can be shared to manage the communication. Devices can be distinguished by address or device ID numbers.

(3) A dynamic link library creates a thread for maintaining communications and command operations of corresponding devices of the dynamic link library.

(4) For TCP / IP connection, the connected state is judged by the Socket state of the device.

(5) For RS485 communication port, the connected state is judged by sending a query frame, then waiting for data to respond, if no reply message received after a given time, then it can be judged that this device has been disconnected, a message will be send to refresh connection status on the software interface.

\section{A. IEC61850 communication adapter}

The hierarchical structure is defined by object-oriented technology. The client reads CID file and parses model to generate MMS objects. CID file can be copied manually and can also be obtained directly from the server. Real-time 
database is generated according to the MMS object parsed.

After the client received the packet, the corresponding data will be put into the real-time database according to MMS objects. ${ }^{[9]}$ If the connection to the server is broken, then repeat step 3 and step 4.

Main modules of IEC61850 client's program is shown in Figure 4.

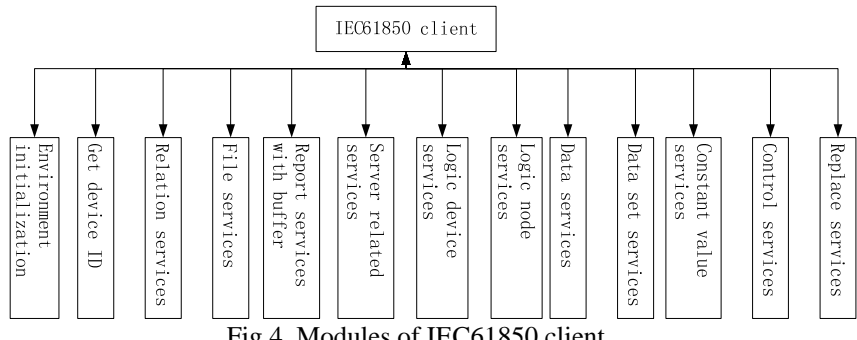

\section{B. Private protocol communication adapter}

Private protocol is mainly referred to those monitoring devices that don't support IEC61850 protocol. To collect the information of sensor connected to these devices, users need to design and develop their special communication protocol adapter for communication with such intelligent monitoring devices.

In the convergence controller layer, all sensor information collected will be modeled uniformly by modeling tools in accordance with IEC61850 protocol and supply for the server to provide unified IEC61850 services.

\section{TYPICAL APPLICATION SCENARIOS}

The typical application scenery of convergence controller are as follows: the sensing devices in substation collect data and store it in the monitoring devices. Different Technologies are used for communication between sensing device and the monitoring device. Monitoring devices transfer data to converge controller by RS-485 serial port or optical fiber; converge controller transfers data to unified access gateway primarily by Ethernet.

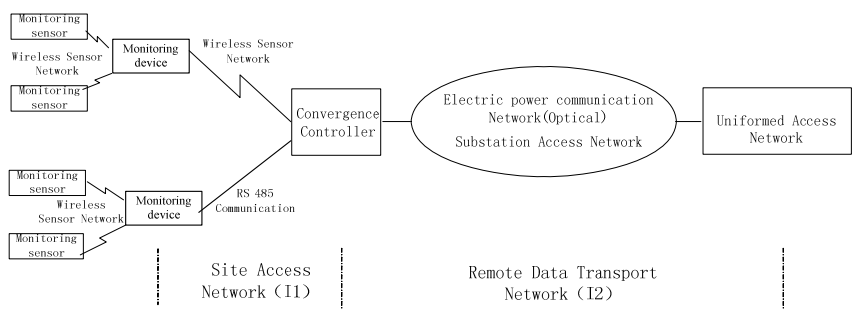

Fig. 5. Convergence communication in substation

In 2012, demonstration applications had been carried out in substation intelligent management according to the overall planning and specific business needs of the power Internet of Things.

Based on the overall framework of the power Internet of Things, demonstration application achieves unified authentications of various contents, including application and testing of convergence controller. Convergence controller, as a primary device which connects with monitoring devices and accesses to gateway equipment uniformly, supplies the sensor data of the perception layer which has been analyzed and processed to the application layer in various forms in order to provide unified data services and unified application services.

\section{CONCLUSIONS}

The convergence controller designed and developed based on IEC61850 protocol in this paper could be applied to the power grid to support safe and reliable information transmission, collaborative processing and unified services, help promote the panoramic awareness, interoperability and seamless integration in the whole process of power grid operation and enterprise management in power generation, transmission, transformation, distribution and consumption processes of smart grid. Sensing data from monitoring devices will be sent to the application layer to be processed and used after analysis, filtering, processing and summary, which will benefit for deepening the power Internet of Things and promoting its applications. ${ }^{[10]}$

With the gradually development and maturing of the Internet of Things technologies, the convergence controller will play an important role to support real-time acquisition, reliable transmission and intelligent processing of the data in power production, operation and controlling, supporting for constructing a strong and smart grid.

\section{REFERENCES}

[1] Li Xiang-zhen, Liu Jian-ming, Zhen Yan, Chen Xi, Zeng Ling-kang. Integration of IoT and smart grid. The 12th Annual Meeting of China Association for Science and Technology (CAST), pp553-558, 2010.

[2] Zhen Yan, Zeng Ling-kang, Chen Xi, Li Xiang-zhen, Liu Jian-ming. Architecture of Power Internet of Things. Electricity, No.5, Vol.22, 2011, pp10-p15.

[3] Liu Jian-ming, Li Xiang-zhen. Internet of Things and Smart Grid[M]. Electronic Industry Press, Beijing, 2012.

[4] IEC61850, Communication networks and systems in substations (First edition) [S]

[5] Wang Wei, Zhu Yong-li. Design and implement of SCL configuration tool, CUS-EPSA 2006, pp125-p128.

[6] Ding Dai-yong, Liu Pin, Wang Xiao-ru. Design and implementation of MMS client software based on IEC61850, RELAY, 35(8), 2007, pp45-49, 73.

[7] Wang Shu-jing, Ye Shen-rui, Zhang Le. Analysis of Implementing the IEC61850 Model with MMS-EASE Lite. INSTRUMENTATION TECHNOLOGY. (6), 2009.

[8] OGC 07-000, OpenGIS Sensor Model Language (SensorML) Implementation Specification, 2007.

[9] IEEE 1451, Standard for a Smart Transducer Interface for Sensors and Actuators-Network Capable Application Processor (NCAP) Information Mode.

[10] Rao Wei, Ding Jian-yong, Li Rui. Application of Internet of Things Technology in Smart Grid. Central China Electric Power. (24) 2011, pp1-5. 